\section{Swiss Cheese Striatum}

Pasquale F. Finelli, MD'

\section{Keywords}

MR imaging, CT imaging, Virchow-Robin spaces, striatum

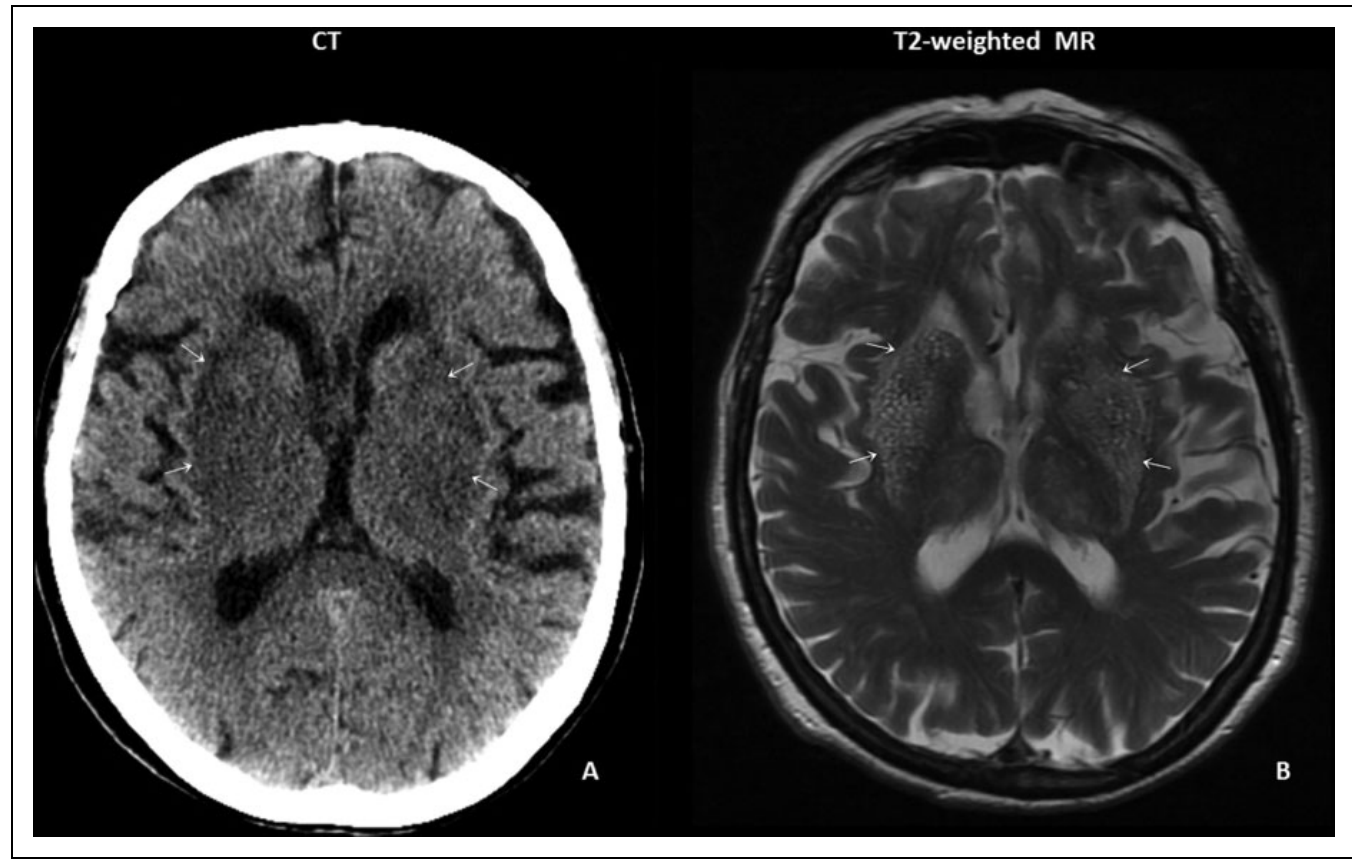

Figure I. Bilateral symmetric decreased attenuation of the striatum on computed tomography (CT; arrows; $A)$ and increased signal of the striatum on T2-weighted magnetic resonance (MR) with numerous, well-defined circular Virchow-Robin spaces isointense with cerebrospinal fluid (arrows; B).

\section{Case History}

A 92-year-old woman presented with new-onset confusion and admission brain computed tomography (CT) showed bilateral symmetric low attenuation of the striatum (Figure 1A). Brain magnetic resonance (MR) showed an area of restricted-diffusion consistent with an acute left posterior cerebral artery territory infarction (not shown), and T2-weighted sequence showed numerous bilateral symmetric small hyperintensities in the striatum (Figure 1B).

\section{Discussion}

Neuroimaging plays a pivotal role in the diagnosis of deep gray matter lesions, and when the striatum is selectively involved, the etiologies include toxic-metabolic, hypoxic- ischemic, vascular, and infectious conditions. ${ }^{1}$ Bilateral symmetric low attenuation of the striatum on CT imaging can be problematic considering multiple diagnostic entities manifest with an almost identical imaging appearance. In such cases, MR can help clarify the diagnosis. In our patient, "Swiss Cheese Striatum" (SCS), an uncommon, benign developmental condition, was diagnosed by characteristic MR imaging features of numerous Virchow-Robin perivascular spaces. ${ }^{2}$

\footnotetext{
' Department of Neurology, Hartford Hospital, University of Connecticut School of Medicine, Hartford, CT, USA

Corresponding Author:

Pasquale F. Finelli, Hartford Hospital, 80 Seymour Street, Hartford, CT 06 102 , USA.

Email: pfinell@harthosp.org
} 
The MR lesions are isointense with cerebrospinal fluid on all sequences, lack enhancement, and are small, sharply defined with circular perimeters. The SCS should be considered among conditions that manifest with bilateral symmetric striatal hypodensity on CT and can be confirmed with MR imaging.

\section{Declaration of Conflicting Interests}

The author declared no potential conflicts of interest with respect to the research, authorship, and/or publication of this article.

\section{Funding}

The author received no financial support for the research, authorship, and/or publication of this article.

\section{References}

1. Finelli PF, DiMario FJ. Diagnostic approach in patients with symmetric imaging lesions of the deep grey nuclei. Neurologist. 2003; 9(5):250-266.

2. Burnett M, Witte J, Ahlskog JE. Swiss Cheese Striatum. JAMA Neurol. 2014;71(6):735-741. 